\title{
The Behavior of Dihydropyrazine with DNA Strand-Breakage Activity in Vivo
}

\author{
Tadatoshi Yamaguchi, ${ }^{*, a}$ Hajime Nomura, ${ }^{b}$ Kazuhisa Matsunaga, ${ }^{c}$ Shigeru Ito, ${ }^{d}$ Jiro Takata, ${ }^{c}$ and \\ Yoshiharu KARUBE ${ }^{c}$ \\ ${ }^{a}$ Department of Hygiene, Miyazaki Medical College; and ${ }^{b}$ Department of Laboratory Medicine, Miyazaki Medical \\ College; Kiyotake-cho, Miyazaki 889-1692, Japan: ${ }^{c}$ Faculty of Pharmaceutical Sciences, Fukuoka University; Fukuoka \\ 814-0180, Japan: and Institute of Biomaterials and Bioengineering, Tokyo Medical and Dental University; Tokyo \\ 101-0062, Japan. Received June 20, 2003; accepted July 15, 2003
}

\begin{abstract}
The effects on the viability of cell lines treated with 2,3-dihydro-5,6-dimethylpyrazine and its derivatives, which revealed DNA strand-breakage activity by the generation of radicals in vitro, were recognized from certain morphological changes and the detection of apoptosis-related proteins: cleaved PARP and SAPK/JNK. These results would suggest that sugar-derived dihydropyrazines induce changes in the cells of certain organs and cause various internal injuries in vivo. The biodistribution of ${ }^{14} \mathrm{C}$-labelled 2,3-dihydro-5,6-dimethylpyrazine was studied in mouse and the autoradiograms showed highly contrasting results. Radioactivity was high in the brain, spinal cord, salivary gland, and thymus and low in the heart, stomach, and blood. The result was supported by the activity (\% dose per organ).
\end{abstract}

Key words dihydropyrazine; apoptosis; biodistribution; DNA strand breakage; cell line; aminosugar

In our previous paper, ${ }^{1)}$ we showed that dihydropyrazines (DHPs) such as 2,3 dihydro-5,6-dimethylpyrazine (DHP-1) revealed DNA strand-breakage activity. The reaction species at that time were presumed to be hydroxyl and carbon-centered radicals ${ }^{2)}$ generated from DHPs in the scission reaction system.

The DHPs were produced primarily from sugar in vitro, namely, the reaction of a carbonyl group of glucose (sugar) with an amino-group of amino acid (protein) to give an amadori rearrangement compound via a Schiff base. The process was followed by Strecker degradation to $\alpha$ aminoketone, including only aminosugars ${ }^{3)}$ which revealed DNA strand-breakage activity, that is, DHPs were produced from two molecules of the $\alpha$-amino-ketone by dehydration. ${ }^{4}$ It is predictable that the formation of DHPs in vitro also occurs in vivo under non-enzymatic or enzymatic conditions. An enzymatic formation ${ }^{5)}$ in vivo of glucosamine-6-phosphate which revealed stronger activity ${ }^{3)}$ of DNA strand scission than glucosamine, is already known. Therefore, if the resultant glucosamine underwent bimolecular condensation, DHPs might be easily produced and show more intensive activity.

On the other hand, a number of pyrazine derivatives, which were readily produced from the dihydropyrazine ring structure by oxidation ${ }^{6}$ (dehydrogenation), were detected in human urine $^{7)}$ and foods. ${ }^{8-10)}$ Thus, it is strongly suggested that DHP as a precursor of pyrazine was formed and found in vivo. We will try to shed light on the effects of DHPs in vivo in this report.

\section{MATERIALS AND METHODS}

Chemicals DHPs (Fig. 1) used in an annexing experiment with cell lines were all synthesized by the condensation of diketones and diamines under the methods in appended papers. 2,3-Dihydro-5,6-dimethylpyrazine (DHP-1), 2,3-dihydro-2,5,6-trimethylpyrazine (DHP-2) and 3-hydro-2,2,5,6tetramethylpyrazine (DHP-3) were synthesized by the method of Yamaguchi et al. ${ }^{1)} 2,4 \mathrm{a} R^{*}, 7,9 \mathrm{a} S^{*}$-Tetramethylcy- clohexano-[1,2e : 4,5e']-dipiperazine-6-ene (DHP-4) was synthesized by the method of Yamaguchi et al. ${ }^{11)}$ Bovine liver catalase (40000 unit/mg protein) and other chemicals were purchased from Wako Pure Chemical Ind., Ltd. used in the molecular biological experiment using cell lines are mentioned below.

Cell Culture and Treatment with DHPs Human HeLa cells were grown as monolayer cultures in RPMI 1640 medium supplemented with $10 \%$ fetal bovine serum. Rat fibroblast 3Y1, NRK49F and mouse teratocarcinoma P19 cells were maintained in Dulbecco's modified Eagle's medium (DMEM) supplemented with $10 \%$ fetal bovine serum. All cell lines were grown at $37^{\circ} \mathrm{C}$ in a humidified atmosphere of $5 \% \mathrm{CO}_{2}$ until $70-80 \%$ confluence was reached. For examination of the effects of DHPs on cells, the cells were incubated with $1 \mathrm{~mm}$ of DHP in culture medium for the indicated times. Photographs were taken by microscope (DIAPHOT TMD, Nikon) to assess cellular morphological changes.

Immunoblotting The cells exposed to DHPs were collected to prepare cell extracts for the following analysis. After being washed two times with cold PBS, the treated cells were scraped and collected through centrifugation. For immunoblotting assay, cell pellets were lysed with sodium dodecyl sulfate sample buffer containing $62.5 \mathrm{~mm}$ Tris- $\mathrm{HCl}$ (pH 6.8), 2\% w/v sodium dodecyl sulfate (SDS), 10\% glycerol, $5 \% \quad \beta$-mercaptoethanol, and $0.1 \% \mathrm{w} / \mathrm{v}$ bromophenol blue. Cell lysates were sonicated in an ice bath for $10-15 \mathrm{~s}$, boiled for $5 \mathrm{~min}$, and centrifuged at $14000 \boldsymbol{g}$ at $4{ }^{\circ} \mathrm{C}$ for $5 \mathrm{~min}$.<smiles>[R]C1([R])CN=C(C)C(C)=N1</smiles>

DHP-1: $\mathrm{R}_{1}=\mathrm{R}_{2}=\mathrm{H}$ DHP-2: $\mathrm{R}_{1}=\mathrm{CH}_{3}, \mathrm{R}_{2}=\mathrm{H}$ DHP-3: $\mathrm{R}_{1}=\mathrm{R}_{2}=\mathrm{CH}_{3}$<smiles>CC1CNC2(C)CC3=NC(C)CN[C@]3(C)CC2=N1</smiles>

DHP-4
Fig. 1. Some DNA Breakable Compounds 
Proteins in the supernatant of each extract were resolved by SDS-polyacrylamide gel electrophoresis (PAGE), and transferred onto Immobilon P membranes (Millipore) in Tris/ glycine transfer buffer. For the detection of proteins, the following antibodies were used; goat polyclonal anti-cadherin, - $\beta$-catenin, and -actin antibodies were obtained from Santa Cruz Biotechnology (Santa Cruz, CA, U.S.A.). Rabbit polyclonal anti-cleaved PARP and -phospho SAPK/JNK antibodies were purchased from New England Biolabs Inc. (Beverly, MA, U.S.A.). The protein/antibody complexes were reacted with alkaline phosphatase-conjugated secondary antibodies (anti-goat and anti-rabbit, Promega) and visualized by color reaction with NBT (nitro blue tetrazolium) and BCIP (5brom-4-chloro-3-indolyl-phosphate) (Sigma, Oakville, Ontario, Canada). The inhibition of DHP activity on cells by radical scavenger was estimated by reduction of cleaved PARP protein, which was calculated as described in Table 1.

Preparation of a Solution of ${ }^{14} \mathrm{C}$-Labeled-2,3-dihydro5,6-dimethylpyrazine ( ${ }^{14} \mathbf{C}$-DHP-1) $10 \mu \mathrm{Ci} / \mathrm{ml}$ for Autoradiography: According to the literature method, ${ }^{1)}$ reaction of ethylenediamine (ED) with 2,3-butanedione (BD) in $\mathrm{D}_{2} \mathrm{O}$ was performed, and the sole product was confirmed to be 2,3-dihydro-5,6-dimethylpyrazine (DHP-1) by NMR spectrographic analysis. After that, ${ }^{14} \mathrm{C}-\mathrm{ED} \cdot \mathrm{HCl}$ instead of $\mathrm{ED}$ was reacted with BD. First, a water solution $(0.1 \mathrm{ml})$ of ${ }^{14} \mathrm{C}$ $\mathrm{ED} \cdot \mathrm{HCl}$ was dehydrochlorinated by the addition in small excess of Amberlite IRA-400. To the $\mathrm{HCl}$-free solution of ${ }^{14} \mathrm{C}$ $\mathrm{ED}$, a $0.4 \mathrm{ml}$ solution of the mixture of ED $0.1 \mathrm{ml}$ and dist. $\mathrm{H}_{2} \mathrm{O} 9.9 \mathrm{ml}$, and dist. $\mathrm{H}_{2} \mathrm{O} 0.494 \mathrm{ml}$ were added. Under cooling on an ice bath, $\mathrm{BD} 6.1 \mu \mathrm{l}$ was added to the mixture and the reaction mixture was allowed to stand for $20 \mathrm{~min}$ on the ice bath under stirring. The resulting reaction mixture of $0.1 \mathrm{mCi} / 0.1 \mathrm{ml}$ of ${ }^{14} \mathrm{C}$-DHP-1 was injected into the tail vein of ICR mice weighing $25-30 \mathrm{~g}$.

For Autoradiography: The mice were killed by ether at 30 and 60 min after injection, rapidly frozen at $-80^{\circ} \mathrm{C}$ and em-

Table 1. Inhibition Effect of Enzyme and Radical Scavengers on Apoptosis Induced by Dihydropyrazine Derivatives

\begin{tabular}{|c|c|c|c|c|}
\hline DHP & Conc. & Inhibitors & Conc. & $\begin{array}{c}\text { Inhibition } \\
\text { rate }(\%)\end{array}$ \\
\hline 3 & $1 \mathrm{~mm}$ & $\mathrm{DMSO}^{a)}$ & $100 \mathrm{~mm}$ & 40.5 \\
\hline 2 & $1 \mathrm{~mm}$ & & & 79.7 \\
\hline 1 & $1 \mathrm{mM}$ & & & 67.4 \\
\hline 3 & $1 \mathrm{~mm}$ & $\mathrm{MEA}^{b)}$ & $5.0 \mathrm{~mm}$ & 79.9 \\
\hline \multirow[t]{3}{*}{3} & $1 \mathrm{~mm}$ & $\mathrm{AET}^{c)}$ & $2.0 \mathrm{~mm}$ & 90.4 \\
\hline & & & 5.0 & 67.2 \\
\hline & & & 10 & 80.9 \\
\hline \multirow[t]{3}{*}{3} & $1 \mathrm{~mm}$ & Thiourea & $2.0 \mathrm{~mm}$ & 59.6 \\
\hline & & & 5.0 & 69.7 \\
\hline & & & 10 & 54.0 \\
\hline \multirow[t]{2}{*}{3} & $1 \mathrm{~mm}$ & $\mathrm{KI}$ & $2.0 \mathrm{~mm}$ & 56.2 \\
\hline & & & 5.0 & 50.6 \\
\hline \multirow[t]{3}{*}{3} & $1 \mathrm{~mm}$ & Catalase & $1.0 \mathrm{mg} / \mathrm{ml}$ & 62.0 \\
\hline & & & 2.0 & 71.5 \\
\hline & & & 4.0 & 63.2 \\
\hline
\end{tabular}

The HeLa cells were incubated with $1 \mathrm{~mm}$ DHP at $37^{\circ} \mathrm{C}$ in RPMI 1640 medium supplemented with $10 \%$ FBS in the presence of enzyme or radical scavenger for $24 \mathrm{~h}$. Inhibition of cellular effects was calculated as follows: inhibition rate $(\%)=\mathrm{Di} / \mathrm{Dc}, \mathrm{Di}$ is the density of PARP protein band in immunoblot of the samples with the scavenger, Dc that of a control without an inhibitor. The measurement of density of the PARP band was done using image analysis software; NIH-image. $a$ ) DMSO: Dimethylsulfoxide, $b$ ) MEA: 2-Mercaptoethylamine hydrochloride, c) AET: $S$-(2-Aminoethyl) isothiouronium bromide hydrobromide. bedded in carboxymethylcellulose. Twenty micrometers serial sections through the sagittal plane of each animal were made by the tape-sectioning method with a Cryo Polycut cryostat (Reichert-Jung, Nussloch, Germany) at $-20^{\circ} \mathrm{C}$. The sections on adhesive tape (Salotape R150, Hisamitsu Pharmaceutical Co., Saga, Japan) were desiccated and placed on an imaging plate (Fuji Photo Film Co., Ltd.) for $1 \mathrm{~d}$. Completed macroautoradiograms were photographically processed using a Bio-imaging Analyzer BAS2000 (Fuji Photo Film Co., Ltd.).

For Estimation of Accumulation: The mice were killed by collection of blood from the heart at 30 and $60 \mathrm{~min}$ after injection. The organs or tissues were resected and weighed. The carbon-14 radioactivity of some organs and blood, which were prepared with a sample oxidizer (model 307, Packard Instruments Company), was counted in a liquid scintillation counter. The percentages of the injected dose per organ (ID/organ) were determined (mean \pm S.D., $n=4$ ) by the ratio of organ radioactivity to the total radioactivity.

\section{RESULTS}

The effects of DHPs on cellular viability were examined by the assay of several cell lines exposed to DHPs. Microscopic photographs of the assay using HeLa cells are shown in Fig. 2. The strength of the morphological changes were in the order of DHP-3 $>$ DHP-4=DHP-2 $>$ DHP-1. After a few hours of treatment with DHPs, morphological changes were detected in the HeLa cells used in the assay, that is, the cells after $24 \mathrm{~h}$-incubation lost cell-cell adhesion, detached from the substrate as pieces of sheets, or changed into a spindle shape in the presence of the DHPs such as DHP-4, -3 and -2. The apoptotic morphological changes of cytoplasm retardation and nuclear condensation were recognized as shown in Fig. 2. However, DHP-1 showed no microscopic changes on treatment at a concentration of $1 \mathrm{~mm}$. Similar results were obtained from assays using other cell lines: 3Y1, P19 and NRK49F.

It was investigated by molecular biological methods whether a cell death pathway was actually induced in DHPtreated cells with morphological changes. ${ }^{12)}$ The cleavage of PARP protein $(89 \mathrm{kDa})$ was detected in the cells treated with DHP-2 and 3, as shown in immunoblotting data (Fig. 3). In addition, the detection of PARP-cleavage was consistent with the time course of the cell morphological changes accompanying decrease in cell viability. However, DHP-1 with weak DNA-cleavage activity did not cause any significant morphological changes that appeared in apoptotic cells; slight PARPcleavage was observed at a concentration of $1 \mathrm{~mm}$.

However, addition of $5 \mathrm{~mm}$ DHP-1 caused similar morphological changes as in the case of DHPs (DHP-2, -3 and -4). It seemed that DHPs according to the order ${ }^{1)}$ of strength of DNA cleavage might cause apoptosis in the treated cells, which resulted in the cleavage of PARP. ${ }^{13)}$

Furthermore, we undertook examination to clarify the pathway of signal transduction from DHPs to PARP-cleavage. When HeLa cells were treated with DHPs, phosphorylation of SAPK/JNK protein was specifically observed in DHP-3 treated cells (Fig. 3). Using rat 3Y1 and NRK cells, remarkable phosphorylation of SAPK/JNK protein was also detected in the cells treated with DHP-2 and 4, as well as 

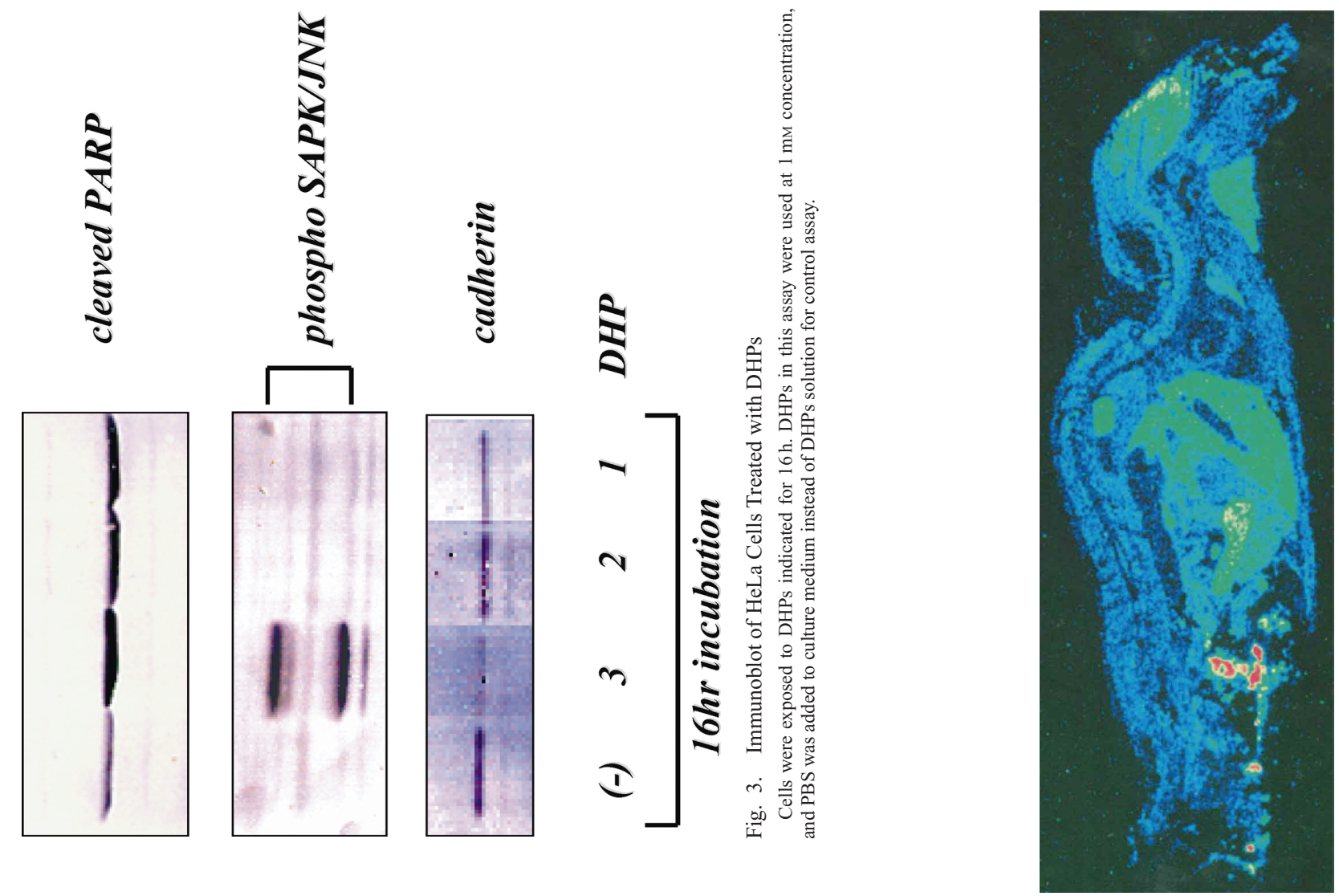

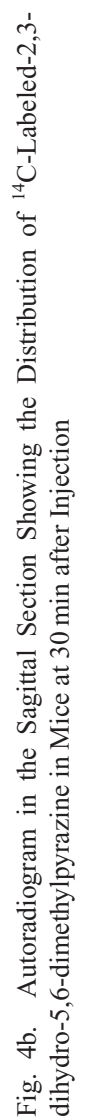
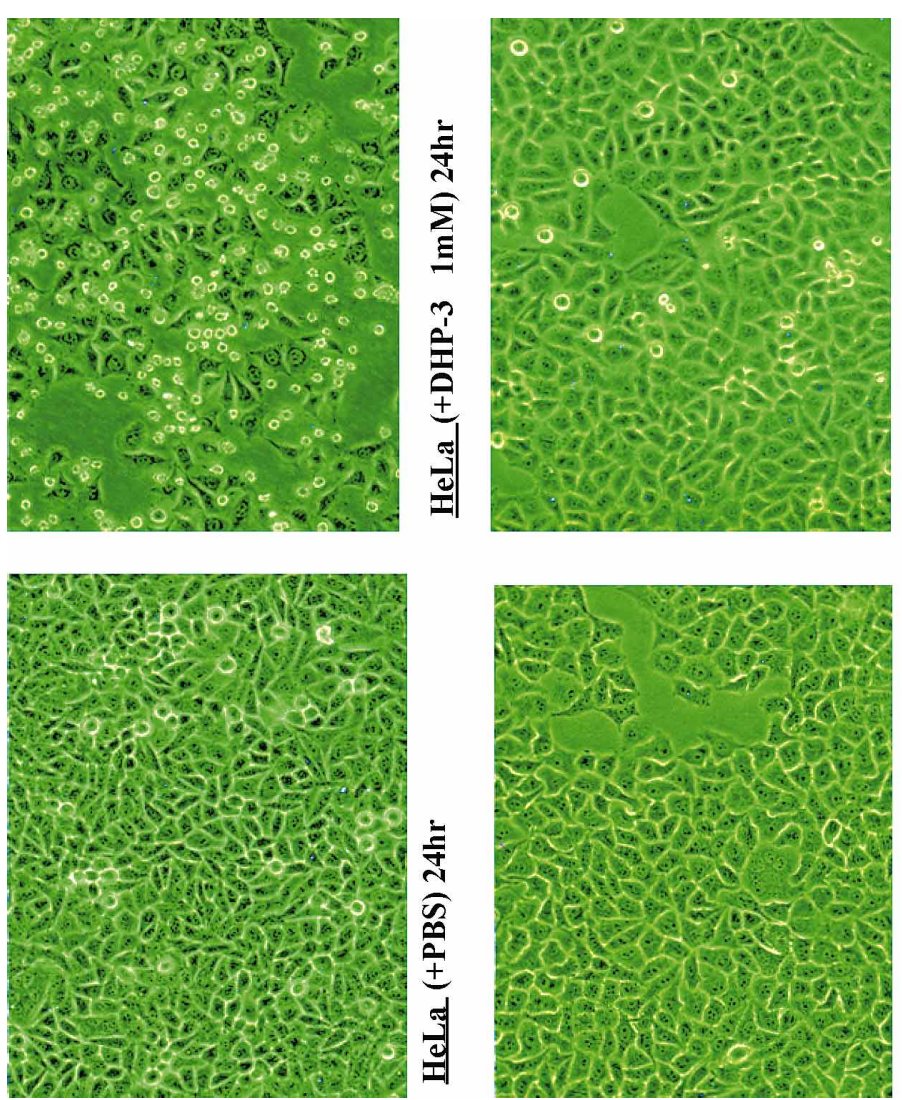

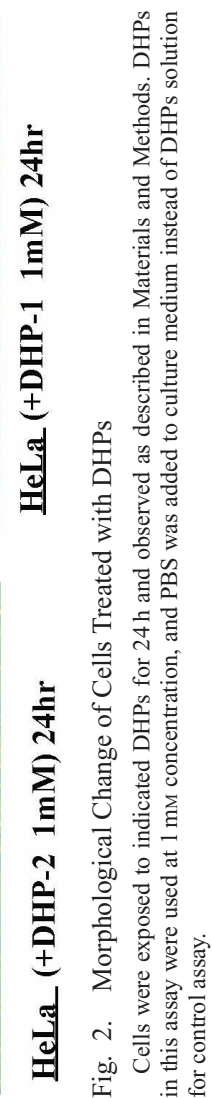

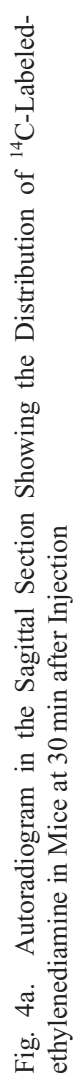


DHP-3. The marked phosphorylation of the SAPK/JNK protein occurred with a dose dependency similar to the case for PARP cleavage and reduction of cell viability. The phosphorylated SAPK/JNK indicated activation of the signal pathway against cellular stress. These results suggest that the stimulation of cells by DHPs results in the stress pathway proceeding with SAPK/JNK activation, which, in turn, might amplify a subset of cascade signals that cause apoptosis.

The order of the strength of the morphological changes is parallel to that of the DNA strand-breakage activity. ${ }^{1)}$ Thus, it was predicted that activation of the signal cascade followed by morphological changes and the apoptosis were due to radicals generated from DHPs. To learn the possible involvement of radicals on apoptosis induced by DHPs, the inhibition effects of catalase and some radical scavengers on DHP activity were examined.

The levels of apoptosis induced by DHPs were estimated on the basis of the density of the cleaved PARP protein band in immunoblotting data (Fig. 3). The inhibition effects (Table 1) were clearly recognized by the addition of various inhibitors. Scavengers of $\cdot \mathrm{OH}$, such as dimethyl sulfoxide (DMSO), thiourea and KI effectively inhibited the production of cleaved PARP protein. Catalases, which cause disproportion of $\mathrm{H}_{2} \mathrm{O}_{2}$ to yield $\mathrm{H}_{2} \mathrm{O}$, also inhibited the apoptosis strongly. The apoptosis was also inhibited more effectively by 2-mercaptoethylamine hydrochloride (MEA) and $S$-(2aminoethyl) isothiouronium bromide hydrobromide (AET), which are well known to scavenge not only active oxygen radicals, but also carbon-centered radicals $(\cdot R)$. These results indicated that the reactive species on apoptosis induced by DHPs were hydroxyl and carbon-centered radicals. ${ }^{2)}$ It is predictable that DNA strand-breakage might be caused in a cell nucleus by the attack of the radical species from DHPs. We attempted to detect the DNA ladder in the cells treated by DHPs, however, it was not possible. Since apoptosis without participation of DNA cleaving was reported, ${ }^{14,15)}$ our results might be the same case. Also, morphological changes, such as loss of cell adhesion and detachment from the substrate, were observed in DHP-treated cells (Fig. 2). We examined the changes in expression of three proteins related with cellcell interaction; cadherin, $\beta$-catenin, and actin. Although the apoptosis was induced, expressions of actin and $\beta$-catenin were not changed after treatment with DHPs. But cadherin, in the case of DHP-3 only, was destroyed after exposure to DHPs as shown in Fig. 3.

Figures 4-a and 4-b show the autoradiogram of ${ }^{14} \mathrm{C}$-labeled-ethylenediamine $\left({ }^{14} \mathrm{C}\right.$-ED) and ${ }^{14} \mathrm{C}$-labeled-2,3-dihydro-5,6-dimethylpyrazine $\left({ }^{14} \mathrm{C}\right.$-DHP-1), respectively. In contrast to ${ }^{14} \mathrm{C}$-ED used in the control experiment, high uptake of ${ }^{14} \mathrm{C}$-DHP-1 was observed in the spinal cord as well as the brain. High uptake in the salivary gland and thymus was also observed.

This result is noteworthy, because it was reported in a previous paper ${ }^{16)}$ that large amounts of glucosamine-6 phosphate were synthesized in the salivary glands and exported to other organs in vivo; thus it is predictable that DHPs are formed vigorously in the gland and have a strong affinity for this gland. Analogous to ${ }^{14} \mathrm{C}$-DHP-1, ${ }^{14} \mathrm{C}$-labeled-2,3-dihydro-5,6-diethylpyrazine, which was synthesized by a similar method, also showed an approximate distribution pattern in mouse. This indicated that a peculiar affinity for the nerve
Table 2. The Distribution of ${ }^{14} \mathrm{C}$-Labeled-2,3-dihydro-5,6-dimethylpyrazine in Whole Body of Mice

\begin{tabular}{|c|c|c|c|c|}
\hline \multirow{3}{*}{ Organ } & \multicolumn{4}{|c|}{ Duration of time after injection } \\
\hline & \multicolumn{2}{|c|}{$30 \mathrm{~min}$} & \multicolumn{2}{|c|}{$60 \mathrm{~min}$} \\
\hline & $\%$ dose $/ g$ & $\%$ dose/organ & $\%$ dose $/ g$ & $\%$ dose/organ \\
\hline Stomach & $2.0 \pm 0.2$ & $0.95 \pm 0.05$ & $2.35 \pm 0.05$ & $0.75 \pm 0.05$ \\
\hline Pancreas & $4.8 \pm 0.3$ & $1.1 \pm 0.1$ & $4.85 \pm 1.35$ & $1.45 \pm 0.15$ \\
\hline Liver & $8.5 \pm 0.0$ & $14.85 \pm 0.35$ & $6.2 \pm 0.2$ & $10.8 \pm 0.0$ \\
\hline Kidneys & $12.35 \pm 0.65$ & $7.5 \pm 0.3$ & $10.55 \pm 3.05$ & $6.8 \pm 1.8$ \\
\hline Spleen & $10.65 \pm 0.95$ & $0.95 \pm 0.05$ & $8.1 \pm 0.1$ & $0.7 \pm 0.0$ \\
\hline Salivary glands & $4.95 \pm 0.95$ & $1.4 \pm 0.3$ & $3.75 \pm 0.05$ & $0.95 \pm 0.05$ \\
\hline Brain (cerebrum) & ) $9.2 \pm 0.6$ & $2.1 \pm 0.2$ & $8.3 \pm 1.9$ & $2.1 \pm 0.4$ \\
\hline
\end{tabular}

center was due to the ring skeleton of dihydropyrazine, although the reason is not yet known. Table 2 shows the radioactivity ( $\%$ dose $/ g$ and $\%$ dose/organ) in each organ at 30 and $60 \mathrm{~min}$ after injection. The values of \% dose/organ were supported by the autoradiogram. High activities were observed in the kidneys, spleen, brain and liver. Especially in the brain, the cerebrum showed higher activity than the cerebellum and others. The activity of the cerebrum at 30 and 60 min was $2.1 \pm 0.2$ and $2.1 \pm 0.4 \%$ dose/organ, respectively. Except for the cerebrum and pancreas, the activities of the other organs decreased with the passage of time over $30 \mathrm{~min}$. The data indicated that the metabolism of ${ }^{14} \mathrm{C}-\mathrm{DHP}-1$ proceeded for the period. However, the activity in the cerebrum was unchanged and that in the pancreas was increased at $60 \mathrm{~min}$ rather than that at $30 \mathrm{~min}$ after the injection.

\section{DISCUSSION}

As mentioned in the introductory paragraph, the formation of DHPs derived from sugars (glucose) in vivo is predictable, especially when excessive glucose exists in the blood. Approximately 40 urine samples from subjects with diabetes mellitus were studied, and a high concentration of pyrazines, which were easily produced from DHPs by oxidation, was found. ${ }^{4)}$ This result indicated that DHP, which was a precursor of the pyrazines, might be related to diabetes mellitus, although it is still unconfirmed whether DHPs cause symptoms of the disease or not. Our previous results in connection with DHPs have suggested their relation with certain diseases. In this paper, we elucidated that DHPs induced apoptosis as suspected from the cleavage of PARP protein in DHP-treated cells and autoradiograms of ${ }^{14} \mathrm{C}$-DHP-1 in mice exhibited as accumulation in organs such as the brain, spinal cord, salivary gland, thymus and liver. These results suggested serious implications as mentioned below. The order ${ }^{1)}$ of DNA strandbreaking activity was DHP-3 $>$ DHP- $2=$ DHP- $4>$ DHP -1 . This order was compatible with the degree of the effect in morphological changes as shown in Fig. 2. In ESR spectroscopy, ${ }^{2)}$ all compounds (DHP-1, -2, -3 and -4) revealed approximately the same signal's pattern due to DMPO-adducts $(\cdot \mathrm{OH}, \cdot \mathrm{OOH}$ and two kinds of carbon-centered radicals), with various levels of intensity. But the DBNBS-adducts spectra showed an alternative signal's pattern, which was due only to certain carbon-centered radicals but not to the agreement between DHP-1 and DHP-2. The DBNBS-adducts were assigned as secondary and tertiary carbon-centered radicals. ${ }^{2)}$ Thus far, various characters of DHPs in vitro have been re- 
ported. In this paper, the role of DHPs in vivo is confirmed.

DHPs having DNA strand-breakage activity were accumulated in certain organs, and then certain radicals generated from DHPs in situ induced the apoptosis of the cells on the organs. The treatment by DHPs with strong DNA-cleavage activity results in rapid reduction in cell viability, as assessed by PARP cleavage, while the effect of DHPs with weak cleavage activity appeared as a late response. It was estimated that the difference of cellular responses against DHPs (as morphological changes and the activation of apoptoticmarker proteins) might be due to the different species of radicals generated from each DHP, because the several species in the radicals are due to the chemical structure of DHPs. In the initial attempt, $1 \mathrm{~mm}$ of DHPs was identified as causing the cleavage of PARP protein, with a significant loss of cell viability. Later, it was also revealed that the effect of DHPs was dose-dependent. Increased concentrations of DHPs caused enhanced effects in the rate of activation of SAPK/JNK that was accompanied by cleavage of PARP. These results suggest that DHPs stimulate primary signal(s), leading to morphological changes in the cells. It was possible that DHPs might activate caspases through signal cascades containing cleavage of PARP, resulting in the reduction of cell viability. Cellular toxic activity of DHPs occurs via several different mechanisms of which radical-induced damage seems to be critical for human health.

DHP treatments resulted in morphological change and PARP-cleavage accompanied by the activation of SAPK/JNK in all cells assayed. But the degree of SAPK/JNK activation by each DHP varied among several cells used in the assays. This result indicated that human cell lines represent specific sensitivity against DHPs differing from the cells derived from another species. DHPs had been implicated in the regulation of signal transduction during apoptotic cell responses, such as PARP-cleavage and SAPK/JNK phosphorylation. To gain further evidence that DHPs act on members in another cell signaling pathway, study was needed on whether they might directly or indirectly act on apoptosis-related proteins that function in several pathways. Taken together, it should be elucidated whether DHPs might regulate cellular functions in vivo.

It seemed that a cell adhesion system containing cadherin, $\beta$-catenin, and actin was not clearly affected by DHPs in this study. So, detailed assay to investigate the effect of DHPs on signal transduction mediated with the cell adhesion system was needed. The precise mechanism of the cellular effects of DHPs is unknown at the present stage but will be studied in our laboratory. As DHPs could be generated as sugar derivatives during metabolic processes in vivo, an understanding of their effects on organs and tissues is important. Additional studies are needed to elucidate the precise mechanisms for DHP-mediated pathogenesis of several diseases. DHPs would be quite useful for pursuing the molecular mechanism of cell damage caused in the diseases like diabetic nephropathy and neuropathy. Furthermore, clear evidence for the relationship of DHPs with pathogenesis should be obtained. DHPs would also be of advantageous in diagnosing the treatment of diseases with cell destruction.
This study has suggested that DHPs can be constantly generated as sugar-derivatives through a metabolic pathway and has revealed a critical role for their apoptotic activity. In other words, the cells of the central nervous system and other organs in which DHPs are distributed may be caused various internal injuries in vivo by the DHPs. The inhibitory effects of tumor growth by glucosamine and mannosamine were already reported. ${ }^{17-20)}$ These effects were thought to be connected with the behavior of DHPs, because some DHPs were easily formed from these aminosugars in vitro. ${ }^{4)}$ It was also reported that a dihydropyrazine (DHP) derivative, 3,6-dihydropyrazine-2,5-dipropanoic acid, ${ }^{21)}$ derived from 5-aminolevulinic acid, caused the increased frequency of liver cancer by DNA damage. ${ }^{22}$

Continuing research of the biological activity of DHPs is in progress.

Acknowledgments This work was partially supported by two Grants-in-Aid for Exploratory Research and Scientific Research (B) from the Japan Society for the Promotion of Science (JSPS).

\section{REFERENCES AND NOTES}

1) Yamaguchi T., Kashige N., Mishiro N., Miake F., Watanabe K., Biol. Pharm. Bull., 19, 1261-1265 (1996).

2) Yamaguchi T., Matsumoto S., Watanabe K., Tetrahedron Lett., 39, 8311-8312 (1998).

3) Kashige N., Yamaguchi T., Ohtakara A., Mitsutomi M., Brimacombe J. S., Miake F., Watanabe K., Carbohydr. Res., 257, 285-291 (1994).

4) Kashige N., Yamaguchi T., Mishiro N., Hanazono H., Miake F., Watanabe K., Biol. Pharm. Bull., 18, 653-658 (1995).

5) Kornfeld S., Kornfeld R., Neufeld E. F., O'Brien P. J., Biochemistry, 52, 371-379 (1964).

6) Manley C. H., J. Food. Sci., 39, 73-76 (1974).

7) Zlatkis A., Bertsch W., Lichtenstein H. A., Tishbee A., Shunbo F., Anal. Chem., 45, 763-767 (1973).

8) Maga J. A., Sizer C. E., J. Agric. Food Chem., 21, 22-30 (1973).

9) Koehler E. P., Manson M. E., Newell J. A., J. Agric. Food Chem., 17, 393-396 (1969).

10) Koehler E. P., Odell G. V., J. Agric. Food Chem., 18, 895-898 (1970).

11) Yamaguchi T., Eto M., Harano K., Kashige N., Watanabe K., Ito S., Teterahedron, 55, 675-686 (1999).

12) In unpublished data, we found some new results that DHPs performed as an activator of MAP kinase. Thus, the cell death pathway on apoptosis in our results was not a simple but a complicating feature.

13) It is difficult to distinguish clearly the resultant quantity of cleaved PARP between DHP-2 and DHP-1. However, we can state that DHP-2 showed a stronger effect than DHP-1 by other MAP kinase (p38 and ERK) (our unpublished data).

14) Itoh T., Nakamura M., Yagi H., Soga H., Ishii T., Handbook of Exp. Pharmacol., 142, 375-397 (2000).

15) Sakahira H., Enari M., Ohsawa Y., Uchiyama Y., Nagata S., Curr. Biol., 9, 543-546 (1999).

16) Tsuiki S., Miyagi T., Adv. Enzyme Regul., 15, 35-52 (1977).

17) Questtel J. H., Cantero A., Nature (London), 171, 252-254 (1953).

18) Bekesi J. G., Molnar Z., Winzler R. J., Cancer Res., 29, 353-359 (1969).

19) Molnar Z., Bekesi J. G., Cancer Res., 32, 380-389 (1972).

20) Onoda T., Norikawa S., Harada T., Suzuki Y., Inoue K., Nishigami K., Cancer Res., 42, 2867-2871 (1982).

21) Bunke A., Zerbe O., Schmid H., Burmeister G., Merkle H. P., Gander B., J. Pharm. Sci., 89, 1335-1341 (2000).

22) Teixeira P. C., Onuki J., Medeiros M. H. G., Dornemann D., Mascio P. D., Biol. Chem., 382, 913-918 (2001). 